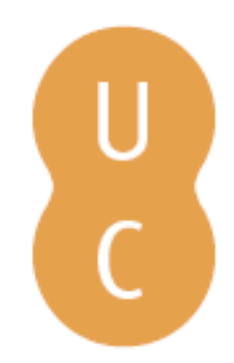

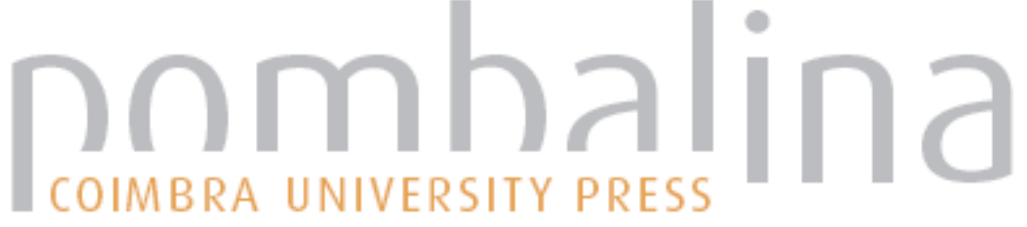

Matter and selfhood in Kant's physics: a contemporary reappraisal

Autor(es): $\quad$ Lori, Nicolás F.; Jesus, Paulo

Publicado por: Imprensa da Universidade de Coimbra

URL

persistente: URI:http://hdl.handle.net/10316.2/31636

DOI: $\quad$ DOI:http://dx.doi.org/10.14195/978-989-26-0205-9_13

Accessed : $\quad$ 26-Apr-2023 01:17:56

A navegação consulta e descarregamento dos títulos inseridos nas Bibliotecas Digitais UC Digitalis, UC Pombalina e UC Impactum, pressupõem a aceitação plena e sem reservas dos Termos e Condições de Uso destas Bibliotecas Digitais, disponíveis em https://digitalis.uc.pt/pt-pt/termos.

Conforme exposto nos referidos Termos e Condições de Uso, o descarregamento de títulos de acesso restrito requer uma licença válida de autorização devendo o utilizador aceder ao(s) documento(s) a partir de um endereço de IP da instituição detentora da supramencionada licença.

Ao utilizador é apenas permitido o descarregamento para uso pessoal, pelo que o emprego do(s) título(s) descarregado(s) para outro fim, designadamente comercial, carece de autorização do respetivo autor ou editor da obra.

Na medida em que todas as obras da UC Digitalis se encontram protegidas pelo Código do Direito de Autor e Direitos Conexos e demais legislação aplicável, toda a cópia, parcial ou total, deste documento, nos casos em que é legalmente admitida, deverá conter ou fazer-se acompanhar por este aviso. 
Edmundo Balsemão Pires

Burkhard Nonnenmacher

Stefan Büttner-von Stülpnagel

Editors

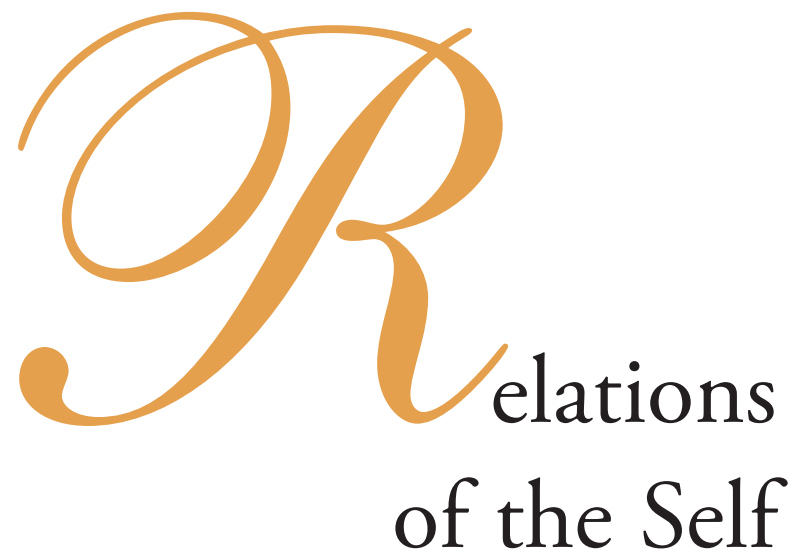


Nicolás F. Lori

IBILI, Faculty of Medicine, University of Coimbra, Coimbra, Portugal. ${ }^{1}$

Paulo Jesus

Center of Philosophy, University of Lisbon, Lisbon, Portugal.

\section{MATTER AND SELFHOOD IN KANT'S PHYSICS: A CONTEMPORARY REAPPRAISAL}

\section{Kant's search for unity}

The son of Werner Heisenberg and nephew-in-law of Carl Friedrich von Weizsäcker, the neurobiologist Martin Heisenberg ${ }^{2}$, who has worked extensively on the neurogenetics of Drosophila, has recently proposed that quantum physics and behavioral biology can solve the Kantian third antinomy in which Reason (Vernunft) appears to be hostage of an insurmountable conflict with itself concerning the contradictory coexistence of two types of causality, namely "causality in accordance with laws of nature" and "causality through freedom" (A444/B472 ff.) ${ }^{3}$. To be sure, the novelty of Heisenberg's proposal does not reside in the idea that the third antinomy is solvable, since for Kant the antinomy is nothing but an illusory contradiction that can be easily "dismantled" and avoided by distinguishing appearances or phenomena (mere representations) that are embedded in an empirically conditioned series in time and space from things in themselves whose intelligible being is situated outside any sensible conditions and enjoys total inde-pendence of all empirical laws. Thus, for him, if the Antinomy is truly understood, then its antinomic structure is dissolved. Indeed, Kant admits that both the realm of empirical causality, which guarantees the unity of experience, i.e. a necessary chain of time-determined occurrences, and the realm of intelligible causality, which constitutes the power of beginning an original action from oneself, can coexist effectively, although in different levels of reality as it were. Both models of causality are "true at the same time but in a different relation".

By using this strategy of differentiation within cognitive functions, between what is determinable by another being (inside temporal relationships as a cause that is, in indefinitum, an effect of a previous cause) and self-determining (outside temporal constraints as a cause that produces itself $a b$ ovo), Kant acknowledges the true effectiveness of the privileged but unknown level of being-in-itself whose key feature is spontaneity - the thing that appears in time and space but remains beyond its appearing. However, under a Kantian

\footnotetext{
${ }^{1}$ To whom mail should be addressed: nflori@fmed.uc.pt

${ }^{2}$ M. Heisenberg, “Is free will an illusion?”, in: Nature, vol. 459, 14 May 2009, p. 459-460.

${ }^{3}$ I. Kant, Critique of Pure Reason, transl. P. Guyer \& A. Wood, New York 1998, p. 484-550.
} 
angle, spontaneity amounts to self-determination (or a self-ruled power), not to indetermination. His determinist framework is absolutely strict and he concedes no room for any randomness or for a breaking of a fundamental state of law, even freedom is always conceived as the ability to generate lawfulness, and thereby to act according to one's own law of action. In a sense, the rationality and the legality of being are the first foundation and the first unquestionable assumption of Kant's critical philosophy, as if it were the conditio sine qua non or the ground-belief of any possible act of thinking and knowing in general. For, even if the "inner legislation" of noumena remains and must remain essentially unknown and unknowable, Kant cannot conceive any being whose behavior is "without reason". Despite his profound construal of spontaneity, Kant does not endorse any absolute form of unpredictability and incomputability, two of the strongest assumptions of contemporary science. Instead, he holds fast to a nomothetic system in such a way that the emergence of possibility and generativity is, perhaps paradoxically, anchored in inner or outer determination, which entails a form of necessity that, in the peculiar case of freedom, exhibits the efficacy of selfnecessitating reasons. It follows that unpredictability should be reducible to the finitude of our understanding and invite us to "epistemic humility" ${ }^{4}$, whereas quantum physics envisages it rather as intrinsic to things and confronts us with an abyssal gap between the spontaneity of being and the traditional belief on its lawfulness or algorithmic regularity.

Moreover, under a Kantian view, freedom begins with the production and adoption of reasons or laws and this process in itself is not necessitated, it is rather a primal force of being. Freedom, nevertheless, opens up immediately a system of self-necessitation, since freedom is the self-gift of Reason, and therefore the suspension of Reason could never be free, but the surrender to the brute causality of lifeless or selfless nature. In fact, just as the antinomy of nature and freedom concerns two kinds of (fully determined) causality, so too the "illusory" antinomy of the faculty of judgment, which divides it between two opposing maxims, two regulative principles, that is, the maxim of mechanical laws and the maxim of teleological laws, is located within the general jurisdiction of determinism ${ }^{5}$. Kant declares non-intelligible the harmonization of these two forms of causality unless one postulates a supersensible being or level of being, being-in-itself, in which organizational ends and efficient causes are perfectly united in a manner that we, humans, have neither concepts nor images to represent it. This transcendental postulation of the systematic unity of both causalities in being or nature and of both maxims in Reason, unity which functions as the ultimate law and as the asymptotic goal for our rational action, is doubtlessly a necessary presupposition for the rationality of all human cognitive endeavor and for the possibility of knowledge ${ }^{6}$. So, in the last analysis, all Kantian dualistic ontological and epistemo-logical distinctions are to be resolved in the transcendental unity of a well articulated and all-encompassing system, where Reason spouses being and nature: "For the law of Reason to seek unity is necessary, since without it we would have no Reason, and without that, no coherent use of the

\footnotetext{
${ }^{4}$ R. Langton, Kantian Humility. Our Ignorance of Things in Themselves, Oxford 1998.

${ }^{5}$ I. Kant, Critique of the Power of Judgment, tr. P. Guyer \& E. Matthews, New York 2002, \$. 69-70.

${ }^{6}$ P. Guyer, "Kant's Principles of Reflecting Judgment", in: P. Guyer (Ed.), Kant's Critique of the Power of Judgment. Critical Essays, Lanham 2003, p. 1-61.
} 
understanding, and, lacking that, no sufficient mark of empirical truth; thus in regard to the latter we simply have to presuppose the systematic unity of nature as objectively valid and necessary." The idea of system means chiefly an articulated whole. This entails a governing principle of organic wholeness translated into a formative and creative force (Bildungstrieb or Erzeugungs- and Bildungskraft), implying the ability of self-organization, that is to say, a strong self-contained reversibility or circularity between cause and effect, part and whole, and means and ends ${ }^{8}$. A "system" stricto sensu is an individual living being, whereas the "system" lato sensu comprise the whole history of life and even the whole cosmic nature, beyond the fracture that divides nature into matter and life.

In Kant's works, the quest for unity, of which the idea of system constitutes the ultimate form, pervades all layers of nature from physics to metaphysics. Thus, in guise of a reconstructive account of Kant's critical construal of systematic unity, let us recall that, according to the First Analogy of Experience (A182/B224 ff.) ${ }^{9}$, the unity of nature lies in the formal unity of experience, that is, the unity or oneness of time, represented by "the identity of the substratum in which alone all change has a thoroughgoing unity" (A186/B229). This means the sameness of a "persisting substance": "the real in the appearance" or "the object itself", "the substance (phaenomenon)", which is the substratum for all time-determinations or the condition of all possible relations and transitions. Although this mode of time, which is "persistence" or "permanence", could be considered a sort of absolute time that comprises all relative times, it cannot be perceived in itself by the inner sense, since in time alone one perceives nothing but succession, and consequently it requires the perception of bodies in space by external senses. But external senses can be affected only by movement, without which there is no knowledge at all of matter. Therefore, Phoronomy, as the physical study of movability, becomes the groundwork of physics (hence the first chapter of the Metaphysical Foundations of Natural Science ${ }^{10}$ ). The a priori unity of nature, disclosed by Phoronomy, is the unification of movement by an immovable absolute space that encompasses all movable relative spaces and makes movement intelligible; for it provides the movable with an ultimate and stable self-referential referent:

Since I have the enlarged, although still always material, space only in thought, and since nothing is known to me of the matter that designates it, I abstract from the latter, and it is therefore represented as a pure, nonempirical, and absolute space, with which I compare any empirical space, and in which I can represent the latter as movable (so that the enlarged space always counts as immovable). ${ }^{11}$

A most difficult unanswered dilemma subsists regarding the physical function of the socalled "nothingness" of absolute space: is absolute space in Phoronomy a mere subjective form or is it rather a material but empty space? In other words, does its nothingness pertain

${ }^{7}$ I. Kant, Critique of Pure Reason, tr. P. Guyer \& A. Wood, New York 1998, p. 595.

${ }^{8}$ I. Kant, Critique of the Power of Judgment, tr. P. Guyer \& E. Matthews, New York 2002, \$. 64-66.

${ }^{9}$ I. Kant, Critique of Pure Reason, tr. P. Guyer \& A. Wood, New York 1998, p. $299 \mathrm{ff}$.

${ }^{10}$ I. Kant, "Metaphysical Foundations of Natural Science", in: I. Kant, Theoretical Philosophy after 1781, tr. H. Allison, P. Heath, G. Hatfield \& M. Friedman, Cambridge 2002, p. 194-208.

${ }^{11}$ I. Kant, "Metaphysical Foundations of Natural Science”, in: Ibid., p. 195. 
to an ens imaginarium or does that nothingness derive simply from the indeterminacy of its matter? Now, given that, in the Metaphysical Foundations of Natural Science, Kant identifies matter with motion and absolute space with a motionless infinite unity, space is then quite problematically severed from matter, as though they were qualitatively heterogeneous concepts - the unity of the first belonging to logical universality and that of the second to physical universality. As a result, the unity of natural science will oblige Kant to think of a stronger form of beingness that be able to replace "absolute time" by elevating matter from motion (in relative movable spaces) to self-motion (in all real space). It is understandable, as formulated in the Opus Postumum, that the passage from metaphysical principles to physics is achieved by the assumption of a materia primitiva movens, which fulfils thereby a selffoundational function as the auto-nomous ontological location of spontaneity. The logical absurdities resulting from the inaccurate notions of void space and separate atoms as well as those of absolute beginning/cessation and an isolated prime mover are avoided by the presupposition of a spatial continuum of formless but spontaneous matter furnishing material for all bodies and agitating them from within.

The proposition: "There are physical bodies" presupposes the proposition: "There is matter whose moving forces and motion precedes the generation of a body in time". For this latter is only the formation of matter, and occurs of its own accord (spontaneo). This formation, however, which is to be initiated by matter itself, must have a first beginning whose possibility is, indeed, incomprehensible, but whose originality (as self-activity) is not to be doubted. Thus there must exist a matter which, as internal, penetrates all bodies (as onus), and, at the same time, moves them continually (as potential). It amounts to a whole which (as a self-subsistent cosmic whole) is internally self-moving and serves as the basis of all other movable matter. [...]

There follows this a priori valid proposition [...]: "There exists a matter, distributed in the whole universe as a continuum, uniformly penetrating all bodies and filling [all spaces] (thus not subject to displacement). Be it called ether, or caloric, or whatever, it is no hypothetical material (for the purpose of explaining certain phenomena, and more or less obviously conjuring up causes for given effects); rather, it can be recognized, and postulated a priori, as an element [Stück] necessarily belonging to the transition from the metaphysical foundations of natural science to physics."12

This primordially self-moving matter could be not only the ultimate warrant for the systematic unity (and autarky) of nature but also the potential physical correlate of the supersensible unity between life and matter. What is more, it might offer the crucial common element or power allowing a physical and metaphysical passage from matter to life. In the Critique of the Power of Judgment (1790), such passage is always declared incomprehensible. In this regard, even the most promising pages of the third Critique $\left(\$ .80-81^{13}\right)$, in which Kant announces the possibility of understanding nature as a unified whole, following the metaphors of genealogy and archeology that imply a perfect commonality of origin, appeals to the supersensible instance of being-in-itself to reconcile the causality of motion with the

\footnotetext{
${ }^{12}$ I. Kant, Opus Postumum, tr. E. Förster \& M. Rosen, Cambridge 1995, p. 68-70.

${ }^{13}$ I. Kant, Critique of the Power of Judgment, tr. P. Guyer \& E. Matthews, New York 2002, p. 286 ff.
} 
causality of generation (without envisaging a truly phenomenal or quasi-phenomenal state of affairs as a self-sufficient ground for both, like the "primordially self-moving matter" in the Opus Postumum). Remarkably enough, those paragraphs (\$. 80-81) propose firstly that, through comparative anatomy and the analogical study of living forms, one can firmly assume an "effective universal kinship" tying them together, and suppose their generation from a "common original model" (Urbild) or "original mother" (Urmutter) - presaging Darwin's "tree of life". Secondly, the underlying principle of continuity among all living forms is applied to the generation of biological novelty, in such a consistent way that Kant considers schematically different types of generation (generatio aequivoca versus univoca, and homonyma versus heteronyma) and examines some defining features of the intra-, inter- and trans-specific generative solutions that will be worked out in detail by Lamarck ${ }^{14}$ and Darwin ${ }^{15}$. Thirdly, Kant extends the principle of continuity and communality from the various kingdoms of organic beings to the domain of lifeless matter, in an attempt to unify all expressions of finality and find out the location for the "first production of something containing ends in itself and being intelligible solely through those ends". It is true that brute matter shows some morphogenetic "techniques" (as in the process of crystallization), which could give some hope for an entirely mechanic explanation of self-organized beings, but for Kant the first brute or chaotic matter should already contain all future potentialities or final dispositions of all natural beings. Hence the merging of mechanism with teleology would be postulated $a b$ initio, without clarifying their relationship. In the horizon of biophysics, an "intelligent", primal and all-pervading, substance - the "universal mother" lies dormant until the awakening of final causes inside efficient causes. However, while the idea of a primordial organized finality increases our intelligibility of nature as teleodynamic system, this gain of intelligibility crosses (and must always cross to Kant) the bounds of our possible experience. Within the Kantian framework, there is no immediate empirical access to any kind of self-determined spontaneity, because this carries with itself a temporal discontinuity, a complete breaking of a fundamental condition of experience, that is, the necessary prior-posterior sufficient linkage of determination. Here, the self-referential cycle, through which Reason self-asserts itself, appears flagrantly embedded in both paradox and necessity: in paradox because ultimately it tends to be a mere monological return of Reason on itself and in necessity because there is no possible rationality without such self-cycling.

Now, the novel and provocative character of the above mentioned M. Heisenberg's resolution - certainly the most tragic hubris for orthodox Kantians - of the well-known conflict of Reason with itself, concerning nature and freedom, and mechanism and teleology, lies in his implicit claim that the phaenomenon/noumenon and empirical/transcendental distinction is superfluous and that, consequently, the existence of "self-initiated actions" can be proved inside the world of sense, and can offer thereby an empirical demonstration for the possibility of transcendental freedom, a possibility that Kant considered object of metaphysical dispute and not susceptible to be properly proved. Unlike Kant, Heisenberg deals with metaphysics in the empirical field. From that risky and fruitful transgression it

\footnotetext{
${ }^{14}$ J.-B. Lamarck, Philosophie zoologique, Paris 1809.

${ }^{15}$ C. Darwin, On the Origin of Species by Means of Natural Selection, London 1859.
} 
follows that genuine self-active and self-referential processes seem then to be detectable in the unpredictability or incomputability of quantum particles and in the adaptive behavior of living beings (including unicellular beings). Human free will, the most sophisticated version of "selfhood" that we know, conjoining empirically efficacious spontaneity with rational self-reference, would be the highest manifestation of undetermined self-generated phenomena, not governed by inner laws but by random self-motion, although expressed in apparently regular patterns. Thus, the categories of incomputable possibility and free spontaneity (rather self-possibilitating than self-necessitating) should be recognized as the first ontological categories. Likewise, phaenomena should be regarded as if they were the overt behavior or the positive "realization" 16 of noumenal selves, because there is nothing but multiple phenomenal expressions of spontaneous forces. If, with and against and beyond Kant, one takes into account the ontological import of contemporary science, it is perhaps high time for a methodological transcendental realism and for an ontological monism - articulated with multi-layered phenomenology. The present text intends to explore a kind of post-critical renewal of Kantian aporias in order to melt some of them in the air.

\section{Revisiting Kant's contradiction between free-will and nature}

Kant's contradiction consists in that all consequence has a reason, be it a determining reason, a sufficient reason or an efficient cause; and this contradicts the subjective experience and belief of freedom and spontaneous agency which is almost impossible to deny as my most intimate experience of acting. This contradiction could be battled by defending the non-existence of laws in nature; but to Kant the non-existence of laws in nature would transform all events in mere "strokes of luck", which for him implies the death of natural science and the neglect of all Newtonian scientific achievements already known and universally acknowledged as true by the intellectual community of his time. For Kant, the most respectful concept in science is the concept of law, whereas those of random and chance were associated with the Atomism proposed by Epicurus ${ }^{17}$ and Lucretius ${ }^{18}$ which he considered to be atheistic absurdities.

So there is necessity/determinism and spontaneity in my Self as natural and moral being (that is as both phaenomenon and noumenon) and in organized beings that constitute "natural ends". But, unless one proceeds to a distinction of levels of being, Kant declares non-intelligible (paradoxical or ungraspable) this coexistence of a double regime of causation: the regime of external causes in determinism and the regime of internal causes (self-caused beings) in spontaneous and teleological phenomena. Now, as far as self-reference is concerned, it seems to require a strong circularity of causes and effects within any system so that it can produce "selfhood", which amounts to the performance of self-emergent and self-organized/

\footnotetext{
${ }^{16}$ G. Buchdahl, The Dynamics of Reason. Essays on the Structure of Kant's Philosophy, Oxford 1992.

${ }^{17}$ Epicurus, "Letter to Menoeceus", in The Stoic and Epicurean Philosophers, transl. by C. Bailey, ed. by W. Oates, New York 1940, p. 33.

${ }^{18}$ Lucretius, On the Nature of the Universe, transl. by R. Melville, New York 1997, Book I, v. 419-422.
} 
/organizing functions. For Kant, although they both exist, as it seems to be self-evident by empirical observation of living beings and by pure self-apperception of my volitional acts, one lacks a logical framework to harmonize them. However, if one admits only the reality of one of those causalities, then one suffers from a deep experiential illusion. From a logical and transcendental point of view, this is an unavoidable and unsolvable deadlock.

The establishment of Kant's contradiction includes in itself Kant's rational idea of a system. Our audacious purpose is to describe how contemporary scientific concepts of "system" - as logical and ontological unity - are capable of overcoming some of the difficulties that Kant considered to be insurmountable, and, through that overcoming, dissolve Kant's contradiction. In order to do that, let us begin by stating what Kant meant to be a system:

...the unity of the manifold cognitions under one idea. This is the rational concept of the form of a whole [Form eines Ganzen], insofar as through this the domain of the manifold as well as the position of the parts with respect to each other is determined a priori. The scientific rational concept thus contains the end and the form of the whole that is congruent with it. The unity of the end, to which all parts are related and in the idea of which they are also related to each other, allows the absence of any part to be noticed in our knowledge of the rest, and there can be no contingent addition or undetermined magnitude of perfection that does not have its boundaries determined a priori. The whole is therefore articulated (articulatio) and not heaped together (coacervatio); it can, to be sure, grow internally (per intus susceptionem) but not externally (per appositionem), like an animal body, whose growth does not add a limb but rather makes each limb stronger and fitter for its end without any alteration of proportion. (A832-833/B860-861) ${ }^{19}$

From this patently a priori construal of "system", whose weakness resides precisely in its hyper-formalist and hyper-logicist structure, one must emphasize the fact that Kant's regulative idea of the "form of a whole" implies the endorsement of a principle of entire computability within a system. For, wholeness signifies the overall unity of a common end, through which a priori determination is achieved, allowing an a priori deduction of any unknown part from its articulation with known ones. So, the metaphysical optimism of the idea of system is quite striking, but it accompanies a hard version of determinism, in that it posits the belief on a knowable "structure" (or topological invariance) of the system that once known makes everything else known within it, including the dynamic of its future growth, since it must follow an a priori law of proportion and qualitative sameness. Moreover, the full a priori determination of a whole constitutes a kind of a priori knowledge of its "perfection", that is to say, the logical completeness and self-contained coherence or consistency of a "fullyfunctioning animal body" as it were. Now, the fact that a real system must also inevitably comprise a certain degree of unforeseeable contingency, creativity and singularity is thus greatly overlooked by this idea of system, brazenly attached to "formal necessity" and to epistemic transparency. The remainder of our argument intends to explore this neglected dimension of creative opacity.

In fact, formal a priori necessity pervades all Kantian epistemology. To such an extent that the basis of Kant's "contradiction" is his perception that physics, as "theory of motion",

\footnotetext{
${ }^{19}$ I. Kant, Critique of Pure Reason, tr. P. Guyer \& A. Wood, New York 1998, p. 691.
} 
and the process of generative spontaneity (most notably human free decision-making) are inexorably at odds, since "self-formation" is irreducible to a play of external locomotive forces. To understand Kant's contradiction in the light of contemporary science, it is necessary to find a form of expressing the relationship between fundamental physics and decision-making neuroscience. These two fields of knowledge are so far apart in scale that we prefer to deal with Kant's contradiction on each of the fields separately, by explaining how contemporary science confronts Kant's contradiction, and only after that can we work on the juncture of these two fields. One is entitled to expect, we think, that once the two fields are joined, the contradiction dissolves itself. We start analyzing the relationship between Kant's contradiction and contemporary science by examining the structure of contemporary physics. Kant opposes "blind necessity" (full mechanism and determinism) and "blind contingency" (full randomness), while contemporary science seems to strike a well-founded balance between those two extremes.

\section{Kant's contradiction and contemporary science}

Due to the radical (transcendental) freedom proposed in Kant's perspective, the Physics one needs to consider must be fundamental enough for such a radical freedom to be assessed as phenomenally realizable - which, for Kant, implies to cross the bounds of intelligibility.

Physics has not yet been able to deal with the integration of the quantum and relativistic behavior, hence the difficulty of accurately defining quantum gravity. We consider, therefore, the quantum behavior not at its more fundamental level, but rather at a level simplified enough in order to attain a clear understanding of its structure while still at a fundamental-enough level so that some degree of radical freedom can still be encountered. This level is the level of the information-based approach to quantum mechanics called quantum Darwinism by W. Zurek ${ }^{20}$. Quantum Darwinism has very good capacity for describing the transition between the Newtonian/classical perspective and the Bohrian/ /quantum contemporary perspective.

Now, quantum Mechanics has several aspects that are relevant for the Kantian contradiction. Whereas in a "classical worldview" existence is objective and determinism is absolute, in quantum mechanics, by contrast, determinism and objectivity appear to be reduced. By classical mechanics we mean both Newtonian mechanics and Einsteinian relativistic mechanics as they are both completely objective and deterministic. Quantum mechanics' time-evolution is described by Schrödinger equation, an extension of the Hamilton-Jacobi equation. The Hamilton-Jacobi equation is the wave representation of classical Newtonian mechanics, and it can be considered the mathematical realization of Spinoza's attempt to describe Newtonian mechanics using Huygens's wave dynamics. Given Spinoza's influence on Kantian thought, it is not unrealistic to consider that Kant was also interested in the development of such an

${ }^{20}$ See W. H. Zurek, "Quantum Darwinism and Envariance”, arXiv: quant-ph/0308163v1, 28 August 2003; W.

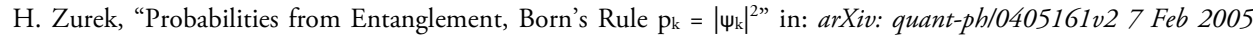
(February 1 2008), and W. H. Zurek, "Relative States and the Environment. Einselection, Envariance, Quantum Darwinism, and Existential Interpretation”, in: arXiv: quant-ph/0707.2832v1, 19 Jul 2007 (February 3 2008). 
approach. The Hamilton-Jacobi equation was first obtained in 1833, and so came too late to help Kant in reassessing Spinoza's physics. In this text, we use the Hamilton-Jacobi equation to detail the alterations to classical mechanics introduced by quantum mechanics.

Classical mechanics can be represented in Newtonian, Lagrangian, and Hamiltonian equivalent mathematical formalisms. In these three mechanisms the system is constituted by particles and it is necessary to explicitly use both the coordinates and velocities of those particles (momentum instead of velocities in the Hamiltonian formalism). It is possible to include waves (also called fields) in those formalisms, but the dynamical equations must always refer to particles. In the Hamilton-Jacobi formalism it is possible to use only waves; the system is represented by two functions, the Hamiltonian $H$ and Hamilton's principal function $S$; and it is possible to use only coordinates $q$ and time $t$, with no explicit mentioning of either velocities or momentum.

The Hamiltonian $H$ is equal to the energy of the system. Hamilton's principal function is the accumulated action from a fixed moment in the past until the present, where the action is simply the difference between the part of the system's energy associated to movement and the part of the energy of the system not associated to movement. The Hamilton-Jacobi equation states that: "the Hamiltonian $H$ is equal to minus the explicit variation of Hamilton's principal function with time". The Hamiltonian in the Hamilton-Jacobi equation depends only on time $t$, the coordinates $q$, and the variation of Hamilton's principal function $S$ with the coordinates $q$.

In quantum mechanics the systems are typically represented by a wave function $\psi$, which assigns a complex number to each coordinate $q$ and time $t$. A complex number is the product of a positive number with a unit size complex number. A unit size complex number can always be represented by the sum of the cosine of an angle with the product of the sine of that same angle with the imaginary unit $i$. The imaginary unit has the properties that its square is equal to "- 1 ", whereas the square of the more typical real numbers are never negative. Complex numbers were first proposed in 1545 by Cardano, the relationship between complex numbers and trigonometry was first obtained by Cotes in 1714, and the geometric significance of that relation was only discovered in 1799 by Wessel but published then only in Danish. The first widely read use of Wessel's results appeared in 1813, again too late for Kant to use it in his approaches to science.

In its coordinate representation form; the Schrödinger equation states that the action of the Hamiltonian on the wave-function equals the imaginary unit $i$ multiplied by the variation of the wave-function with the physics-scaled time. The action of the non-movement part of the Hamiltonian on the wave function is simply a product, but the action of the movement part of the Hamiltonian on the wave-function replaces the wave-function by the mass-scaled double variation of the wave-function with the physics-scaled coordinates. The physics scaling is obtained through the multiplication by a constant called the reduced 
Planck constant $\hbar$, and the mass-scaled alteration is simply the division by twice the mass $m$ of the system. ${ }^{21}$

Schrödinger's equation is presently believed to be valid for all low velocity quantum mechanics, for high-velocity quantum mechanics the used equation is typically Dirac's equation which can be put in a Schrödinger-like form, but whose details are not relevant for the present argument. What is meant by low velocity is a velocity a lot lower than the speed of light in vacuum, and this is almost always the case so the discussion presented here is very general. If the wave-function is divided into its positive amplitude $A$ and its angular phase $\phi$, it is possible to obtain an Hamilton-Jacobi-like equation starting from the Schrödinger equation. The Hamilton-Jacobi-like equation becomes the Hamilton-Jacobi equation if we consider that the reduced Planck constant is almost equal to zero, and that the phase $\phi$ is equal to the physics-scaled Hamilton's principal function $S$. The obtained Hamilton-Jacobi equation does not depend on the positive amplitude $A$, which means that the classical mechanics dynamics depends almost exclusively on the variations of the wave-function's phase. This is related to the square of $A$ being equal to the probability of occurrence of a system's state in quantum mechanics, which has no role in deterministic quantum mechanics. The conversion of a quantum system into an apparently classical system is hence strongly dependent on the relation between the phase and the amplitude of the wavefunction.

In Kant's contradiction a conflict is proposed between the apparently deterministic physics and the apparently non-deterministic human free-will. For a free-will to be able to exist in a world governed by the laws of physics, it is Kant's perspective that the laws of physics have to be not completely deterministic. Quantum mechanics appears to have a certain non-deterministic character; we shall therefore focus on how the transition from classical to quantum mechanics occurs.

To study the transition between quantum and classical mechanics we will use quantum Darwinism. The advantage of quantum Darwinism over other approaches to quantum mechanics is that it does not have a postulate of the existence of a classical world as it occurs in the Copenhagen interpretation, thus allowing for a transition between quantum and classical that is a smooth quantitative transition rather than a sharp qualitative transition. The Copenhagen interpretation is for now the most widely used interpretation despite its several flaws that quantum Darwinism seeks to address. In quantum Darwinism all aspects of the universe are governed by the laws of quantum mechanics. As the name indicates, quantum Darwinism borrows considerably from the concept of natural selection first

${ }^{21}$ The mathematical representation of the Hamilton-Jacobi equation is: $H_{\left(t, \mathbf{q}, \frac{\partial S}{\partial \mathbf{q}}\right)}=-\frac{\partial S}{\partial t}$; and that of the Schrödinger equation is: ${ }^{H}\left(\frac{t}{\hbar}, \frac{\mathbf{q}}{\hbar}, \psi, \frac{\partial \psi}{\partial\left[\frac{\mathbf{q}}{\hbar}\right]}\right)=i \frac{\left(\frac{t}{\hbar}, \frac{\mathbf{q}}{\hbar}\right)}{\partial\left[\frac{t}{\hbar}\right]}$ (or in a more typical but equivalent notation, $\left.H \psi=i \hbar \frac{\partial \psi}{\partial t}\right)$ 
proposed by Darwin in 1859. We will hence analyze quantum Darwinism and relate its structure to the requirements Kant makes about the existence of a system.

According to Zurek ${ }^{22}$, the foundations of quantum Darwinism are the following:

o. The universe consists of systems (the definition of system being that it is individually accessible to measurements).

i. The state of a system is represented by a vector in its vector space, with all vectors in that space having an inner product, the norm of a vector is the square root of the inner product of a vector with itself, and in all series where the norm of a vector joining two vectors goes to zero the two vectors become the same vector.

ii. The time change of a state vector is such that the inner product of a state vector is preserved, which is satisfied by a Schrödinger equation where the matrix representing the Hamiltonian has an imaginary component that is anti-symmetric (trivially true if the Hamiltonian matrix is real); which implies that the time evolution of the system is a unitary transformation.

iii. Immediate repetition of a measurement yields the same outcome.

From the statements $o$-iii two very relevant statements are obtained: the statements iv and $v$ described further below. We first describe the obtaining of statement $i v$, and then proceed to describe the obtaining of statement $v$. Statement $o$ establishes the existence of systems, let us consider system $S$ surrounded by a set of systems that surround $S$ and that we call $\varepsilon$. Henceforth, $S$ will be called "the system" and $\varepsilon$ will be called "the environment". According to statement iii, there must be a set of states of the system that remains unaltered by the interaction with the environment, call it vector state set $S_{k}$. This interaction described in statement iii implements a measurement, so there must be an alteration of the environment leading to the environment being now represented by the vector state set $\varepsilon_{k}$. Due to statement $i$, the state of the system can be represented by a vector state; but due to statement $i i$ the evolution of that state must preserve the inner product and thus also preserve the norm of the state. The joining of statements $o, i, i i$, and $i i i$ implies that the evolution of the state of the system must be such that it is capable of going from a state of not-linked to the environment to a state of being linked to the environment, without altering its norm.

The only way this evolution can happen is if the inner product between any of the vector states of the system that has an influence on the vector states of the environment is equal to zero, no matter how small the influence in the environment. This guarantees that the states of the system capable of leaving a print in the environment, no matter how small, will be orthogonal vector states (two non-zero vectors are orthogonal if, and only if, their inner product is equal to zero). The norm of the orthogonal vector states of the system can be made equal to one, but the clarification of the value of that norm will be assessed when discussing the obtaining of statement $v$. The interaction with the environment makes the states of the system that can transmit information into the environment be orthogonal, but

${ }^{22}$ W. H. Zurek, "Relative States and the Environment. Einselection, Envariance, Quantum Darwinism, and Existential Interpretation”, in: arXiv: 0707.2832v1, 19 Jul 2007. 
once the transmission of information about the system to the environment makes the vector states of the system be orthogonal, the vector states of the environment associated to the corresponding states of the system can themselves be orthogonal. In the case where the environment vector states are themselves orthogonal, the environment is said to have a perfect record of the system.

The orthogonality of the vector states capable of being measured in a certain environment strikes a balance between the interchangeability among states expressed in statements $i$ and $i i$ with the need for a certain state to be able to preserve its existence after measurement expressed in iii. The preservation of the information about the measurement in the environment in large enough quantities to be read by multiple observers after the measurement has occurred is what creates the illusion of state "objectivity" which is a characteristic of classical mechanics. The statement $i v$ thus obtained is, then, as follows:

iv. The measurement outcome of a system is one of the orthogonal unit vector states of the measured observable.

Statement iv is equivalent to the collapse of the wave function postulated in the Copenhagen interpretation of quantum mechanics, except that it does not yet explain the actual collapse, it simply describes that the multiple vector states that can exist after a measurement are orthogonal amongst themselves. In the multiple universe interpretation, each of these orthogonal vector states exist in their corresponding universes, but this interpretation has strong problems involving arbitrary selection of the universe branches in the multi-verse set of universes. The quantum Darwinism interpretation is that the reality of the states is given by their capacity to transmit information about themselves, and that the transmission of that information extinguishes the states which do not succeed in transmitting that information. The successful transmission of information is the printing of the information about the system in the environment states. The Copenhagen interpretation considers that the quantum states are simply epistemic, and attributes an ontic existence only after the collapse of the wave-function. The perspective of quantum Darwinism is that both attributions are excessive. The states before the "collapse" have a certain ontic aspect to them as they are all what the system is before the measurement; and the state of the system after the measurement has a certain epistemic aspect to it as the system does not completely adopt its objective existence, but only does so in as much as it is capable of leaving prints about itself in the environment. The quantum vector states, Zurek explains, are always somewhat epistemic and somewhat ontic, and so he calls them epiontic states.

A fully known (also referred to as pure) entangled combination of system and environment can be described by a vector state where each component of the vector is the product of a system output state $S_{k}$ with its corresponding environment output state $\varepsilon_{k}$. The coefficient associated to that product of output states is called the Schmidt coefficient and is a complex number as it typically happens in vector states. According to statement $o$, the universe consists of systems; statement $i$ makes it explicit that the state vector is the representation of the system; and statement $i i$ describes the change of the state vector of a system in a form that preserves the inner product. Combining the statements $o$-iii it is clear a transformation of a system must act on the vector space of that system to affect the system; the vector space 
of a system larger than $S$ which includes $S$ is all that is needed and available to describe the state of the system $S$; and the state of the system $S$, given the measurements already performed, is all that is needed and available to predict measurement results such as the probabilities of outcomes. The action of a transformation on system $S$ cannot change the output states of either the system or the environment because they were obtained from the process described in statement iv and the transformation cannot change the amplitude of the coefficients because it must be a unitary transformation as described in statement $i$. The same can be said of a transformation acting on the environment $\varepsilon$. This implies that for an entangled system-environment state an action on the system causes an angle shift on the phase of the Schmidt coefficient, and that angle shift can be compensated by an action on the environment. Regularity is an important part of Physics, and if a system is not altered by a certain occurrence the system is said to be invariant under that occurrence. In quantum Darwinism it is possible to describe a kind of invariance where a system is altered by a transformation, but where that alteration can be nullified by an action on the environment, without a further action of the system; and Zurek calls this an environment-assisted invariance, which in short form is called envariance.

If the entangled system-environment state is envariant under a certain transformation acting solely on $S$, then the state of the system $S$ must be invariant under that transformation because the state of a system including $S$ is all that is needed and available to describe the state of the system $S$. Therefore, in an entangled system-environment fully known state, the state of $S$ (or of $\varepsilon$ ) is invariant under changes of the phase of the Schmidt coefficient associated to each outcome state. So the state of $S$ can only depend on the amplitude of the Schmidt coefficients of the outcome state and the outcome state itself. The state of $S$ does not need to be fully known. Decoherence (a.k.a. the collapse of the wave-function) is thus a selective loss of the relevance of phases for the state of $S$, hence a consequence of the onset of envariance, and it is a process that occurs gradually through time as opposed to the drastic change proposed in the Copenhagen interpretation of quantum mechanics.

If outcome states of a system-environment entangled system have the same Schmidt coefficient amplitude, then they are swappable. Because the output states of $S$ and $\varepsilon$ are perfectly correlated one to one, the probability for each of them must be equal. After two outcome states are swapped in $S$ by a transformation not acting on $\varepsilon$ their new probabilities must be the same as the probabilities of their new $\varepsilon$ partners, but the $\varepsilon$ states were not altered since the transformation did no affect $\varepsilon$. So the probabilities of the two $S$ states must be unchanged by the swap, meaning that they must be identical. Thus, probabilities of envariantly swappable states are equal. When all countable $N$ Schmidt coefficients have the same amplitude, the probability of the output states associated to each Schmidt coefficient is the same, and by normalization reasons it is equal to $1 / N$. Associated to each Schmidt coefficient there is a system and an environment output state, which thus have a probability of $1 / N$ of occurring when measured. So, we obtain from statements $o$-iii the statement $v$ below:

v. Probability of finding the state $K$ as the outcome in the measurement of a quantum system prepared in the state $\psi$ is equal to the square of the amplitude of the inner product between the vector state representing state $K$ and the vector state $\psi$. 
The entanglement caused by the Darwinian selection of states that are capable of printing information about them in the environment, creates a loss of importance of the phases of the system which allows for the sum of the probabilities of measurement occurrences instead of the sum of vector states. This occurs because full knowledge about the entangled system-environment state implies complete ignorance about a part of the system and viceversa. In sharp contrast with Kant, one can either know the whole or its parts but not both. The relevance of this relation between the whole and its parts in quantum Darwinism, makes it a relational interpretation of physics interactions. The relational nature of quantum Darwinism renders it easily compatible with the tenets of Einstein's relativity although we did not analyze this in any detail in here.

Lori and $\mathrm{Blin}^{23}$ made an analysis on how a mathematical axiomatic formalism that aimed at completeness rather than consistency (as it is typically done) would need to stop being a formal axiomatic system (FAS) to become a Darwinian axiomatic system (DAS). The axioms of an axiomatic system are complete if all statements expressed in the axiomatic system can be logically valuated given the logical value of the axiomatic system's axioms, and are consistent if for no statement $\alpha$ can both $\alpha$ and not- $\alpha$ be provable from the axioms ${ }^{24}$.

In Leibniz's concept of law the initial number of forms of existence can be smaller than the number of final forms of existence allowed by the law. To describe Leibniz's concept of law one could use the concepts of enablement, alternative and message. The enablement are the rules of inference, and the alternative are the inferred statements. The message has a double role, when the message leaves a system it causes the aspect of the alternative that is incompatible with the message to be extinguished, while the aspect of the alternative that is compatible with the message is maintained. When a message enters a system, it alters the way the enablement will affect the alternative (see Figure 1). The message is the mechanism by which extinction occurs.

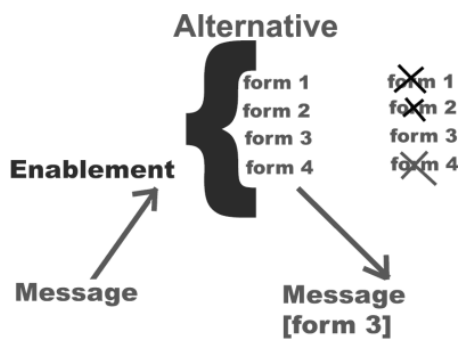

Figure 1: Structure of Leibniz's approach interpreted using the concepts of enablement, alternative and message.

${ }^{23}$ N. F. Lori \& A. Blin, "Application of Quantum Darwinism to Cosmic Inflation: an example of the limits imposed in Aristotelian logic by information-based approach to Gödel's incompleteness", in: Foundations of Science, Special issue on the First International Conference on the Evolution and Development of the Universe, 160-168, 2010.

${ }^{24}$ G. J. Chaitin, Meta Math!: The Quest for Omega, New York 2005. 
For there to be no decoherence, one must look at the whole universe as the system, which would in this case be both deterministic and unmeasurable. If a partial system of the universe is measured, then the output states reveal only a portion of the state of the system but it is exactly that portion that is capable of leaving a print in the environment. The dynamics of the outputted measured values preserves the deterministic aspect of the evolution of the whole universe, and this selection of output state will make all other output states unable to influence the environment, becoming thus de facto extinct. The transition between the FAS of the whole universe which does not have the capacity to leave a print and the FAS of the printed outcome states requires a DAS of selection of which of the outputted states occurs. Given that the decoherence is never absolutely accomplished, and that systems are always a bit decohered (even maybe the whole universe), it is best to represent quantum Darwinism as an hybrid axiomatic system (HAS) combination of a FAS and a DAS ${ }^{25}$.

Kant's contradiction is concerned with the apparent conflict between a deterministic physical world and the intimately felt and believed existence of human free-will. The contemporary approach to the felt/believed freedom of free-will is strongly grounded on Damasio's somatic model of consciousness approach to decision-making ${ }^{26}$, which is strongly based in Spinoza's conatus essendi $i^{27}$. This somatic model of consciousness perspective on feelings is that they are neuronal representations of emotions occurring in the body and that they affect the decision-making process by altering how options will be considered. Under this somatic model of consciousness, feelings are understood as being the result of pain vs. pleasure states of the cells constituting the body (primarily the gut) with pleasure representing homeostatic balance. An alternative to the somatic model of consciousness is one where the neuron's "pleasure" represents the validity of outcome predictions ${ }^{28}$. These outcome predictions refer to outcomes that are not provided by the body, but are rather stored in the expressed part of the neuron's DNA ${ }^{29}$.

Leibniz developed his law in an attempt to allow for compatibility between human freewill and the determinism of Newtonian physics. Contemporary neuro-science obtains that neurons represent the future actions of the perceived entities as if they could choose their actions, implying that perceived entities can be neuronally represented as agents with free-

${ }^{25}$ N. F. Lori \& A. Blin, “Application of Quantum Darwinism to Cosmic Inflation: an example of the limits imposed in Aristotelian logic by information-based approach to Gödel's incompleteness", in: Foundations of Science, Special issue on the First International Conference on the Evolution and Development of the Universe, 160-168, 2010.

${ }^{26}$ A. Damasio, The Feeling of What Happens, New York 2000.

${ }^{27}$ See A. Damasio, Looking for Spinoza, New York 2003.

${ }^{28}$ M. D. Hauser, N. Chomsky, \& W. T. Fitch, "The Faculty of Language: What Is It, Who Has It, and How Did It Evolve?", in: Science, 298, 1569-79, 2002; P. R. Montague \& G. S. Berns, "Neural Economics and the Biological Substrates of Valuation”, in: Neuron, 36, 265-84, 2002.

${ }^{29}$ E. R. Kandel, “The Molecular Biology of Memory Storage: A Dialogue Between Genes and Synapses”, in: Science, 294, 1030-8, 2001; P. Greengard, “The Neurobiology of Slow Synaptic Transmission”, in: Science, 294, 1024-30, 2006; A. J. Pocklington, M. Cumiskey, J. D. Armstrong \& S. G, N. Grant, "The proteomes of neurotransmitter receptor complexes form modular networks with distributed functionality underlying plasticity and behaviour", in: Molecular Systems Biology. Doi: 10.1038/msb4100041, 2006; C. Badcock \& B. Crespi, "Battle of the sexes may set the brain”, in: Nature 454, August 28, 1054-1055, 2008. 
will. This is not sufficient for the affirmation of free-will; it simply affirms the capacity for neurons to represent agents as if they had free-will (including oneself), which is an unchallenged assumption. The objective existence of free-will requires a detailed balance between physics and neuronal decision-making that is presented in a different work ${ }^{30}$, and is outside the scope of this work.

Spinoza believed that the correct interpretation of physics was the wave-like approach proposed by Huygens. This choice by Spinoza is likely to have been motivated by his doing lens work for Huygens. Kant died in 1804 at a time where physics was still dominated by the particle-based approach to physics developed by Newton; for it was only after the 1830's that the wave-based approach developed by Huygens started gaining increasing importance. Both Newton and Huygens were deterministic, but both Spinoza and Leibniz understood the philosophical implications of a deterministic universe and tried to find ways around them. Spinoza proposed the conatus essendi to allow biological organisms to be non-caused generators of their actions, and Leibniz proposed the previously described alternative-generating generalization of Newton's determinism where God is able to intervene by selecting the best of multiple alternatives.

It was proposed here that the mathematically correct form of representing physics and neuronal decision-making is a HAS. In both the quantum and the neuronal case the DAS appears as the mechanism capable of making a transition between FAS levels with different alphabets and grammars. This would at first appear to indicate an opting for dualism, but the choice is neither for an FAS or a DAS but rather for an HAS where the mostly FAS preservation of the effects of internal representations are in continuing relationship with the mostly DAS pressure for the dissipation of internal representation conflicts in order to achieve an agreed external stable representation. No matter if the internal representations are a quantum vector state or expressed genes in the neurons; and if the external stable representation is a measured physical value or a human decision.

\section{Conclusion}

In Kant's contradiction either free-will is an illusion (which would transform man into an amoral being), or causal determinism is not absolute (which would in Kant's opinion make science void). We used axiomatic systems to represent both information-based quantum mechanics and the somatic approach to decision-making. Kant's contradiction combines both perspectives with the result being that they are found to be incompatible.

Now, there are two extreme forms of resolving Kant's contradiction. The first form is to conclude that free-will does not exist, and even cannot exist at all, as conclude both Damasio and Dennett. The second form is to choose a non-entangled, quasi-dualistic, approach where human decision-making is not definable by the laws of physics, and so the causal determinism

30 N. F. Lori, "Relation between Contemporary Physics and Free-will: an Application of Quantum Existentialism”, in: Mind \& Matter, vol. 7 issue 1, 2009, p. 111-129. 
of physics does not affect the existence of free-will. We propose an intermediary form already defended by Lori in a previous work $^{31}$ that the information-based approach to quantum mechanics allows for a compatibilist approach; and we use the axiomatic system perspective of Gödel's incompleteness developed by Lori \& Blin ${ }^{32}$ to more easily establish the link between quantum mechanics and human decision-making.

In Kant's perspective, it is not possible to find an intermediary form between the mentioned two forms. The reason for Kant's perceived impossibility is in our opinion twofold: i. Kant believed in Reason as the basis for his argument, and so he was stuck to a FAS-like approach; ii. Kant was aware of the possibility of a teleological perspective for physical dynamics, but he disagreed with Leibniz about both the ultimate validity and the usefulness of such approaches. It was incomprehensible for Kant how a teleological system (governed by ideal causes) might be fully compatible with a mechanical system (governed by real causes).

Our proposal is that in quantum Darwinism this interaction is possible because of the transcendental randomness ("transcendental" meaning here non-calculable or non-computable) of quantum events as expressed in the experimental breaking of Bell's inequality. A FAS is a logical process, and a DAS is a teleological process where the purpose is universal harmony between propositions. Logic and teleology are hence complementary aspects of axiomatic systems, which can only interact if intrinsic (at once noumenal and phenomenal) randomness exists. Kant did not believe it would be possible for true randomness to exist, but it does exist, as Leibniz expected.

The existence of true randomness allows for the analysis of the quantum systems as systems having true spontaneity: an inner degree of self-creative and self-formative power. Chaitin's information-based approach to Gödel's incompleteness theorems implies that selfreferential axiomatic systems with finite amount of information cannot be both complete and consistent ${ }^{33}$. An always effective FAS cannot be constrained by anything else, so for a FAS to be capable of interacting with a DAS it is necessary that the FAS is capable of noncausal inference (a.k.a. randomness).

Through this transition it is possible a certain degree of freedom that allows one to speak of freedom not only at a fundamental level but also at a level of freedom for a system, where it must be included the language that the system is able to interact with. The neurons are themselves constituted by molecules following the rules of quantum mechanics, and so, if those molecules have in themselves a Kantian noumenal spontaneity, then the ensemble of molecules might also have a Kantian ideal systematic unity. But for a molecular ensemble to have a Kantian systemic selfhood certain characteristics must be fulfilled. The ensemble would need to have oneness, wholeness and teleological unity that requires a kind of continuous self-referential loop; meaning that the system would need to be a DAS. The oneness expressed by the existence of an alphabet-grammar-axioms support system, which allows for

${ }^{31}$ N. F. Lori, "Relation between Contemporary Physics and Free-will: an Application of Quantum Existentialism”, in: Mind \& Matter, vol. 7 issue 1, 2009, p. 111-129.

${ }^{32}$ N. F. Lori \& A. Blin, “Application of Quantum Darwinism to Cosmic Inflation: an example of the limits imposed in Aristotelian logic by information-based approach to Gödel's incompleteness", in: Foundations of Science, Special issue on the First International Conference on the Evolution and Development of the Universe, 160-168, 2010.

${ }^{33}$ G. J. Chaitin, Meta Math!: The Quest for Omega, New York 2005. 
the capacity of the DAS to work as a unit. The freedom at the quantum level is transcendental/ /unconditioned, and so the freedom at the level of the neurons will carry some of that unconditionality ${ }^{34}$; and hence the decision-making at the level of the neurons will be spontaneous. The self-referential spontaneity at the neuronal level, allowed by the specifics of quantum Darwinism, satisfies the necessary conditions for the possibility of free-will. Those necessary conditions might also be sufficient, provided that two characteristics be fulfilled: i. The propagation of randomness is enough for the action of the system to be free; ii. The system generates its own laws of self-development.

To sum up, from quantum mechanics to decision-making by neuronal ensembles there are several orders of magnitude of interactions; and analyzing how those interactions occur is still an open scientific question. In the meanwhile, we are further entitled by this work to reinforce our belief in ourselves as selves.

\section{Future Work}

A more thorough discussion of Kant's contradiction requires an analysis of how the self occurs or is perceived in any act of the human will. For Kant, freedom constitutes/is the production and adoption of reasons or laws, being the self a necessity for the possibility of reasons and laws. If humans have different available levels of self conceptualization, there will be a free-will for each of these levels. Meaning that in Kant's perspective free-will is not only about the attaining of the maxima for each of the self conceptual levels, but also about the attaining of an acceptable dialogue between the different maxima. The relative importance given to each of the maxima would be different for different societies, might even constitute the most essential of the societal differences.

A research direction we are pursuing on the relationship between human will and human self conceptualization consists on relating social emotions with Leibniz's law. The use of Leibniz's law would suggest that three negative feelings correspond to each positive feeling; with each negative feeling corresponding to failure of one of the three components (enablement, alternative, and message) of Leibniz's law in successfully predicting the outcome, while each positive feeling corresponds to a successful outcome prediction. Past and recent experimental evidence has lent support to the idea that different positive social feelings would be associated to differing levels of complexity of self-models ${ }^{35}$. The consciousness levels proposed by Damasio were the proto-self, core self and extended self. Although it is not yet clear what is the relationship between the consciousness levels and the self-model used in perceiving the social feelings, our research direction is considering the possibility that the self-models proposed by Damasio can be further detailed by dividing the extended self into three components: auto-biography, historical-biography and universal-biography self levels. This separation of

34 N. F. Lori, "Relation between Contemporary Physics and Free-will: an Application of Quantum Existentialism”, in: Mind \& Matter, vol. 7 issue 1, 2009, p. 111-129.

${ }^{35}$ A. Damasio, Looking for Spinoza, New York 2003; M. H. Immordino-Yang, A. McColl, H. Damasio \& A. Damasio, "Neural correlates of admiration and compassion", in: PNAS, May12, vol. 106 (19), 2009, p. 80218026. 
the extended self into three levels allows for a direct link between consciousness level and social feelings. The auto-biography self is based on permanent records of memorized coreself experiences and strongly overlaps with Damasio's concept of extended self (in Damasio's work the two names are used interchangeably, we are proposing a differentiation of the autobiographical and extended self concepts); the historical-biography self occurs through the inclusion of stories/histories not directly lived but that are felt to be connected with the person's ancestry; and the universal-biography self is an extension of the ancestry concept to the whole universe and even beyond.

The association between Table 1 and Damasio's social emotions is somewhat straightforward for the successful and de-alternatived columns. For the de-enabled column, embarrassment corresponds to anxiousness (a combination of fear and tension) about the possibility of being surprised again in the future, and shame corresponds to anxiousness about the possibility of becoming an outcast in the future. In the de-alternativated case, the dual role of emotions/ /feelings as describers of situations and as internal normative ethics enforcers is relevant ${ }^{36}$. As for sympathy and compassion, they are pleasant internal normative ethics that aim at counteracting the occurrence of de-messaged feelings in others. Sympathy counteracts feelings of jealousy from others, by highlighting the existence of a certain degree of reciprocity at the level of autobiographical self. Compassion counteracts feelings of being abandoned by others, by emphasizing the existence of a certain degree of reciprocity at the level of historical-biography self. The close correlation between the feeling of hopelessness and depression ${ }^{37}$, hopelessness expressing the feeling that the Universal provides no alternatives, makes depression the emotion/feeling associated with a de-alternativated universal-biography self.

\begin{tabular}{|c|c|c|c|c|}
\hline Consciousness level $\boldsymbol{\uparrow} \| \mathrm{DAS}$ parts $\rightarrow$ & De-enabled & De-alternatived & De-messaged & Successful \\
\hline Universal-biography self feelings & Guilt & Depression & Forsakenness & Awe \\
\hline Historical-biography self feelings & Outcast & Contempt & Abandonment & Elevation \\
\hline Auto-biography self feelings & Surprise & Anger & Jealousy & Pride \\
\hline Core self feelings & Fear & Disgust & Sadness & Happiness \\
\hline Proto-self feelings & Fatigue & Tension & Malaise & Well-being \\
\hline
\end{tabular}

Table 1: Relationship between constituents of Leibniz's concept of law, and human feelings.

Acknowledgment: N. F. Lori and P. Jesus were supported by FCT!

${ }^{36}$ E. Fehr \& S. Gachter, "Cooperation and Punishment in Public Goods Experiments", in: American Economic Review, 90 (4), 980-94, 2000.

${ }^{37}$ L. Y. Abramson, G. I. Metalsky \& L. B. Alloy, "The hopelessness theory of depression: Does the research test the theory?", in: L. Y. Abramson (Ed.), Social cognition and clinical psychology: A synthesis (pp. 33-65), New York 1988. 